\title{
How open science can benefit bilingualism research: A lesson in six tales
}

Rodrigo Dal Ben (dalbenwork@ gmail.com; 0000-0003-2185-8762)

Melanie Brouillard (melanie.brouillard@ mail.concordia.ca; 0000-0002-7129-3221)

Ana Maria Gonzalez-Barrero (anamaria.gonzalezbarrero@mail.concordia.ca; 0000-0002-21206329)

Hilary Killam (hilary.killam@concordia.ca; 0000-0002-3080-0610)

Lena V. Kremin (lena.kremin@ mail.concordia.ca; 0000-0002-4010-490X)

Erin Quirk (erin.quirk@ mail.concordia.ca; 0000-0002-2467-2894)

Andrea Sander-Montant (andrea.sandermontant@concordia.ca; 0000-0001-9732-3460)

Esther Schott (esther.schott@mail.concordia.ca; 0000-0002-0114-4773)

Rachel Ka-Ying Tsui (rachelkytsui@gmail.com; 000-0002-0152-3132)

Krista Byers-Heinlein (k.byers@ concordia.ca; 0000-0002-7040-2510)

\section{Concordia University}

Running head: Open science and bilingualism research

Author note: Correspondence should be addressed to Rodrigo Dal Ben, Department of Psychology, Concordia University, 7141 Sherbrooke St. West, Montreal, QC, H4B 1R6, Canada. E-mail: dalbenwork@gmail.com

Author statement: RDB led the paper, $\mathrm{KBH}$ supervised, and all remaining authors contributed equally.

Conflicts of interest: None.

Acknowledgements: This work was supported by grants from the Natural Sciences and Engineering Council of Canada to KBH (402470-2011; 2018-04390), by a Concordia Horizon Postdoctoral grant to RDB, a fellowship from the Fonds de Recherche du Quebec to LVK and ES, KBH holds the Concordia University Research Chair in Bilingualism and Open Science.

\section{Number of Figures: 0}

Number of Tables: 1

Number of words: 4955 


\section{Abstract}

41 Bilingualism is hard to define, measure, and study. Sparked by the "replication crisis" in the

42 social sciences, a recent discussion on the advantages of open science is gaining momentum.

43 Here, we join this debate to argue that bilingualism research would greatly benefit from

44 embracing open science. We do so in a unique way, by presenting six fictional stories that

45 illustrate how open science practices - sharing preprints, materials, code, and data; pre-

46 registering studies; and joining large-scale collaborations - can strengthen bilingualism research

47 and further improve its quality.

48

49 Keywords: bilingualism, open science, replicability, reproducibility, large-scale collaboration

50

51 
**MANUSCRIPT UNDER REVIEW - FEBRUARY 2022**

\section{How open science can benefit bilingualism research: A lesson in six tales}

Cutting-edge research pushes the frontiers of the unknown and provides more complete scientific explanations of our world (Scheel et al., 2020). At the same time, no matter how impressive or popular a study is, replication by independent researchers is the ultimate test of scientific knowledge (Nosek \& Errington, 2020). In this way, science advances in an interplay of innovation and confirmation, improving with each iteration. However, recent assessments of the scientific literature have revealed that this interplay may be unbalanced (Ioannidis, 2005;

Simmons et al., 2011). Some research findings formerly thought to provide solid evidence have been shown to be hardly replicable, calling established social science phenomena into question (Dailey \& Bergelson, 2021; Ebersole et al., 2020; Klein \& Al., 2013; Open Science Collaboration, 2015).

These findings have sparked productive debates on why such troubling replication failures have happened and how to overcome them (Agnoli et al., 2017; Banks et al., 2016; Bergmann et al., 2018; Frank et al., 2017; John et al., 2012; Leivada et al., 2020; Sijtsma, 2016; Syed \& Kathawalla, 2020; Wicherts et al., 2016). Take-home messages from these debates include a need for embracing transparency throughout the research process, learning about and avoiding questionable research practices, ensuring reasonable statistical power, and collaborating to increase sample size as well as researcher and participant diversity.

This set of practices is commonly known as open science and is increasingly embraced by many disciplines (Nosek et al., 2015). Discussions of open science in bilingualism research in the published literature have been limited (although see Leivada et al., 2020), yet we believe that these practices can be especially beneficial to bilingualism research, in particular by improving 
75 how researchers approach and understand the rich variation inherent to bilingualism (Marian \& Hayakawa, 2020). One example of how open science can bolster bilingualism research comes from Byers-Heinlein and colleagues (2021), as part of the ManyBabies Consortium. The Consortium set out to replicate a robust finding from the developmental literature: infants' preference for infant-directed over adult-directed speech. Using open science practices, 17 labs from 7 countries collected data from 333 bilinguals and 385 monolinguals between the ages of 6 and 15 months and found that both bilinguals and monolinguals showed comparable preference for infant-directed speech versus adult-directed speech. Having such an unusually large sample of bilingual infants also allowed the researchers to demonstrate a continuous relationship between exposure to the stimulus language and the magnitude of infants' preference. Critically, these effects were found after controlling for variance arising from labs and infants, either entering them as random effects in a mixed-effects model, or estimating their variation using a meta-analytic approach, making results more generalizable and helping to disentangle competing explanations. Other researchers have also shown how open science can benefit bilingualism research on more disputed topics, such as bilingual cognitive advantages (Leivada et al., 2020). However, while some bilingualism researchers have begun to adopt open science practices, these are not yet the norm everywhere in bilingualism research. This might be due to lack of awareness as well as real or perceived challenges in changing the way that research is conducted.

We believe that the adoption of and consistent use of open science can lead us to a much stronger science of bilingualism (Ioannidis, 2005; John et al., 2012; Simmons et al., 2011; Wicherts et al., 2016). Yet, the transition to open science can sometimes be fraught with feelings of anxiety and uncertainty. We suggest imagining the researcher who is new to open science as the protagonist at the outset of an adventure story. The tale usually begins with the researcher 
98 safe and warm in the lab, cozying up to their traditional research practices, not knowing that an adventure awaits. But then it happens: a mysterious journal article mentions the words

"Registered Report", an enthusiastic colleague pleads for them to join their own open science journey to pre-register their collaborative study, or perhaps an all-powerful funding agency decrees that open science shall be the rule of the land. Will the researcher brave the unknown and 103 embark on an adventure towards open science practices?

105 embark on the journey and what they might encounter along the way, although it may also be of 106 interest to others who have begun to venture forth but could use a little encouragement. Here, we 107 have drawn from our lab's open science experiences to craft six tales of bilingualism researchers 108 discovering different components of open science: The Legend of the Preprint; A Fairy Tale 109 About Pre-Registration; Open Materials: A Memoir; Open Data and Analysis code: Mystery 110 Solved; An Epic Tale of Large-Scale Collaboration; and The Story of the Adventurous Dr. All-in111 One. For readers who are short on time or less into storytelling, we refer you to Table 1, which 112 summarizes the open science practices illustrated in the stories and provides further readings. For 113 those who do read on, we hope that our stories resonate with your experiences, as they have with 114 our own, and provide you with encouragement to begin or continue your open science adventure. 


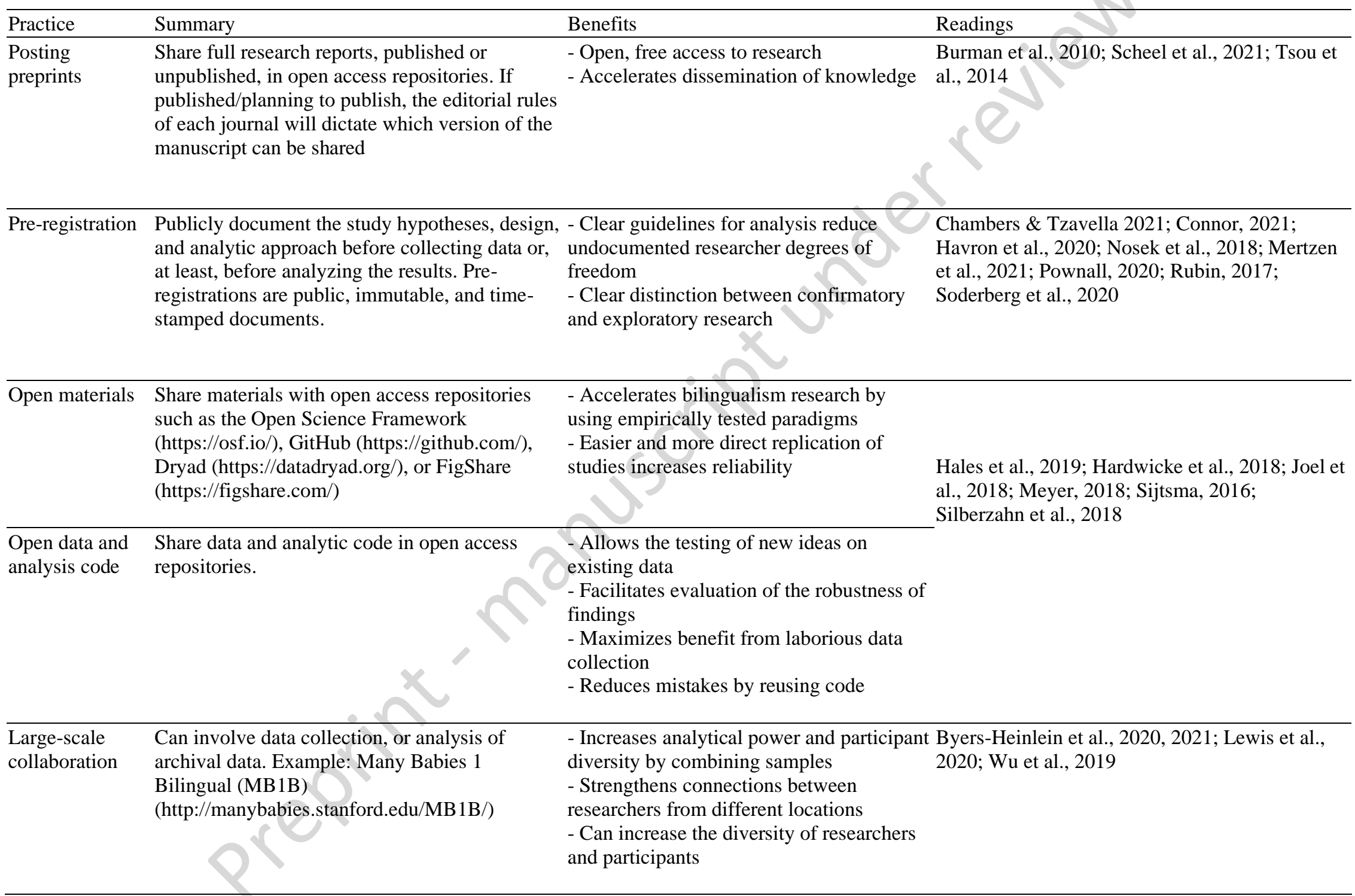


Embrace open Embracing open science is a benefit, not a

science burden. Numerous good arguments have been

presented in this regard. See the column

"Reading" for some suggestions.
Aczel et al., 2019; Allen \& Mehler, 2019; Banks et al., 2019; Crüwell et al., 2019; Frankenhuis \&

Nettle, 2018; Kathawalla et al., 2020; Lindsay,

2020; McKiernan et al., 2016; Munafò et al.,

2017; Murphy et al., 2020; Nelson et al., 2018;

Wicherts et al., 2016; Yamada, 2018 


\section{The legend of the preprint}

Dr. File-Drawer was frustrated. It was a feeling they had experienced before when submitting to their favorite and most well-respected journal in their field, The International Journal of Esperanto and Exercise. This journal was famous for its 17-round peer-review process. Dr. File-Drawer was reading the first round of 7 reviews that each had divergent points

122 of view on their study. The research had compared monolinguals and bilinguals on their ability

123 to learn Esperanto while running on a treadmill. The study was methodologically sound, but

124 there was no difference in performance between the two groups - the dreaded null result. Despite 125 knowing its importance to other researchers - especially for meta-analyses - Dr. File-Drawer

126 feared that it would be months, nay years, before the paper would reach the end of the peer

127 review process, which was particularly arduous for papers that reported null results. And indeed, 128 if Dr. File-Drawer's resolve faltered, this study could end up like so many others in the file 129 drawer.

But this time there was hope. There were legends circulating of a mythical place where research could be shared promptly and openly: The Land of the Preprint. Although this land had odd place names (e.g., PsyArXiv, bioRxiv, arXiv, MetaArXiv), it seemed that anyone around the world, from inside or outside of academia, could enter this land and learn about the latest

134 scientific findings, even before the peer-review process was complete. After a quick search on 135 the internet, Dr. File-Drawer was mesmerized. The Land of the Preprint was real! Wandering 136 throughout this land, Dr. File-Drawer found many interesting projects that they had never seen 137 before, and marveled at such an inclusive, diverse scientific oasis. Indeed, as they explored further, Dr. File-Drawer found one interesting study that had already tested differences between monolinguals and bilinguals in learning Esperanto while swimming, which appeared to also be 
140

141

142

144

under review at the International Journal of Esperanto and Exercise - perfect to cite in their own paper! Looking through the different manuscripts, some were of higher quality than others, but Dr. File-Drawer was able to use an open comments feature to publicly participate in discussions of different papers, and in a few cases they e-mailed the authors to schedule a more in-depth video chat.

Despite the expected years-long wait until publication, Dr. File-Drawer did not feel quite so daunted anymore. Even while the paper underwent the journal's extensive review process, Dr. File-Drawer posted their paper as a citable preprint, uploading updated versions from time to time that incorporated feedback from both peer-reviewers and others who happened upon the preprint. Indeed, Dr. File-Drawer noticed that some researchers posted their preprints for feedback even prior to journal submission.

After the expected 17 rounds of revisions over 7 years, Dr. File-Drawer's paper was finally accepted for publication. As a last step, Dr. File-Drawer uploaded a final version of their manuscript to The Land of the Preprint in accordance with the journal's open-access policies (see https://v2.sherpa.ac.uk/romeo). Dr. File-Drawer kept the copyright to their paper, and scientists around the world were able to read their important work without paying the exorbitant access fees charged by The International Journal of Esperanto and Exercise.

Dr. File-Drawer appreciated the peer review process for the valuable feedback that reviewers provide, and for its role in verifying the scientific quality of contributions prior to their entry into a field's literature, but had always felt frustrated by its glacial pace. At the end, they felt they had the best of both worlds: a peer-reviewed publication in a well-regarded journal, which was also freely available to other researchers as a preprint.

\section{A fairy tale about pre-registration}


Once upon a time, while watching a track and field competition, Dr. Fortune-Teller

164

165

166

167

168

169

170

171

172

173

174

175

176

177

178

179

180

181

182

183

184

noticed that bilingual competitors were unusually likely to win the walking backwards event.

Could monolingual-bilingual differences in walking backwards be a real effect? To test this idea more formally, he designed an elegant study, with rigorous analyses, and happily found that his hypothesis was supported by the data! But reviewers were in disbelief that Dr. Fortune-Teller had accurately predicted the (admittedly unusual) effect he found and suspected he had Hypothesized After the Results were Known (HARKing; Kerr, 1998). Because of these doubts, Dr. Fortune-Teller was unable to publish his groundbreaking findings. He was so downtrodden that he vowed to never let this happen again.

Remembering a piece of advice from his trusted colleague Dr. Early-Bird, an advocate for open science practices such as pre-registration, the next time that Dr. Fortune-Teller made an unusual observation about bilinguals that he planned to test formally (that they could learn Klingon faster than monolinguals), he decided to pre-register his study. Dr. Fortune-Teller wrote up his hypothesis, data collection plan, and data analysis plan as a pre-registration and registered it on the Open Science Framework (https://osf.io). This created an eternal, crystal clear record of his hypotheses, predictions, methods, and data analysis plan, stamped indelibly with the exact moment of its creation. His predictions again proved to be remarkably accurate, and the results of this study supported his hypothesis! This time, when the reviewers questioned whether he had engaged in HARKing, Dr. Fortune-Teller was ready. His pre-registration demonstrated that he had in fact hypothesized the effect before running the study. He also didn't despair when some unexpected findings that even he couldn't predict occurred - he simply wrote them up as exploratory results. Clever Dr. Fortune-Teller! 
Excited about the feedback he was getting on his new publication, Dr. Fortune-Teller reached out to Dr. Early-Bird to thank her for the advice. Dr. Early-Bird was thrilled but then said with a cryptic grin, "But, of course, pre-registration is not the entire story. Registered Reports are also changing research pipelines - [dramatic pause] - they have all the benefits of pre-registration, with the added advantage of a two-stage peer review!"

Dr. Early-Bird went on to explain that in the first stage, reviewers evaluate the research idea and design and eventually grant "in-principle" acceptance. With this initial blessing, the author collects and analyzes the data, then writes the final manuscript. In the second stage of review, the reviewers check everything to make sure the authors followed their original analysis plan, but they also welcome additional exploratory analyses (as long as they are labeled as such). Critically, the statistical significance of the results does not affect whether or not the report is published. Dr. Fortune-Teller could not help but think that the publication bias that had long loomed over bilingualism research would start to dissipate as pre-registrations and Registered Reports became more common. Indeed, Dr. Fortune-Teller's hope was not misplaced. He soon learned that more and more journals in his field are accepting Registered Reports submissions (https://www.cos.io/initiatives/registered-reports) and that despite requiring some up-front preparation, Registered Reports lead to more reproducible and transparent research (Chambers \& Tzavella, 2021). Dr. Fortune-Teller was glad to see that his friend got it right again; open science could indeed lead to a brighter and more reliable science of bilingualism.

\section{Open materials: A memoir}

Future-Dr. Brouillard (yes, a co-author of this paper!) woke up on a beautiful summer morning and decided out of the blue to move data collection for her in-person study online. 
overtaken by a pandemic. Chaos and uncertainty abounded, but one thing remained certain for Future-Dr. Brouillard: data collection must continue.

Though many of the tasks she was using in her experiment were standardized measures, already administered online by other researchers with published results, Future-Dr. Brouillard did not have much luck finding existing code for programming these tasks to be presented online. She felt like she was re-inventing the wheel, having to create most of her tasks from scratch. It took her weeks to record the audio stimuli alone. However, the internet is a big place. After tireless searching, Future-Dr. Brouillard discovered another researcher's previously written code for one of the tasks and was quickly able to adapt it to her needs. Using previously developed (and tested) materials not only saved Future-Dr. Brouillard weeks of work and troubleshooting, but also improved the standardization, and, most likely, replicability of her experimental tasks (Hales et al., 2019). She could be confident her results were comparable to other researchers' without worrying that small differences in stimuli or task presentation were affecting her findings.

In the hopes that it might save other researchers time and effort, as the code she had found had done for her, Future-Dr. Brouillard uploaded her own materials, including code and stimuli, to the Open Science Framework (https://osf.io; see also Brouillard \& Byers-Heinlein, 2021 for the repository mentioned in this tale). Now, should anyone wish to replicate or expand on Future-Dr. Brouillard's work, they could use the very same stimuli that she used and also consult her code to answer any questions not addressed in her manuscript. If, instead, a researcher is studying a population in a different cultural context, Future-Dr. Brouillard's open materials could make it easier to adapt them to be culturally-sensitive resources for another study. Future-Dr. Brouillard would of course be credited for her contributions via citation. 
Future-Dr. Brouillard felt confident that openly sharing materials could make a big

232

233

234

235

236

237

238

239

240

241

242

243

244

245

246

247

248

249

250

251

252

253

difference for the field of bilingualism. For instance, one of the most notorious debates in

bilingualism research regards the very definition of bilingualism (early/late,

simultaneous/sequential, balanced/unbalanced, categorical/continuous...; Kremin \& Byers-

Heinlein, 2021; Surrain \& Luk, 2019). By openly sharing questionnaires and scripts used during

a study, colleagues (or virtual colleagues, to be more exact) could quickly identify which

definition was used to guide inclusion and exclusion criteria. Moreover, they could examine the

effects of using different criteria in analyzing the dataset. In addition, by openly sharing

materials, hidden moderators such as stimuli complexity and differences in task programming

are placed out in the open - topics that are particularly important in bilingualism research. Future

researchers, particularly those interested in directly replicating the original results, could build on

previous work instead of starting from scratch every time, increasing the reliability of

bilingualism science.

After uploading the last of her materials to the Open Science Framework, Future-Dr.

Brouillard sighed contently. Soon those beautiful days when she could run her studies in person

again would return. Whether her studies would be online or in person, she knew that she would always be part of the open materials community, working to create more interpretable, reliable, and replicable research.

\section{Open data and analysis code: Mystery solved}

The Bilingual Research Convention was about to start. Dr. Analysis II, herself fluent in

26 languages, was early, as usual. The eerie light of the vending machine mixed with the smell of

fresh coffee filled the room with a sense of anticipation. While waiting for the talks to start, Dr.

Analysis II headed toward the baked goods, following the unmistakable scent of a fresh cherry 
254 danish. As she reached for a pastry, she caught a glimpse of a poster booth buzzing with activity.

255 The gasps and whispers coming from the crowd became louder as she approached the poster. It

256 all made sense when she read the title: "Bilinguals are 10 times more likely to break their legs: a

257 study spanning 3 skiing seasons" by Dr. Sharemuch and colleagues. She must have spent close to

258 an hour examining the poster in silence. How can this be? she thought. All those years skiing by

259 day and studying bilinguals by night, and I never noticed we were at such risk! The more she

$260 \mathrm{read}$, the more questions she had. The worst part was that the poster author would arrive late to

261 the conference, and two days of waiting was too much for Dr. Analysis II. Enough was enough!

262 Feeling consumed by frustration, something caught her eye. It was a small detail that made a big

263 difference: two colorful badges, the open materials and open data badges, next to a checkered

264 QR code. She was so happy she almost hugged a crowd of undergraduate students who had

265 stopped to peek at the poster everyone at the conference was talking about.

266 After a full day of interesting talks and multilingual networking, Dr. Analysis II rode the

267 crystal elevator towards her hotel room. She was weary, but her thoughts never left that one

268 intriguing poster. She poured herself a glass of white wine and used her laptop to access the

269 website associated with the QR code she had snagged at the poster session. She shrieked in

270 delight as the online repository for the research appeared on her screen. It was better than she

271 could have ever imagined! Row after row of glorious data, and folders containing other goodies:

272 a "readme" file, variable codebooks, and full data analysis scripts.

273 Dr. Analysis II delved into the scripts, looking for clues to explain Dr. Sharemuch's

274 contentious findings. After grasping the main ideas, she downloaded the data and replicated the

275 results with her own analysis script. How could this be? How could bilingualism have anything

276 to do with bone-breaking risk? Driven by curiosity, she delved deeper and deeper into the data 
277 for an explanation. After hours of investigation, long into the night, she found it — a third variable 278 that could explain the pattern! The mystery was solved. Of course, she thought, Bilinguals may 279 break their legs more often, but they also tend to go down harder routes (e.g., black diamonds) 280 than monolinguals! Finally satisfied, she fell asleep.

Two days later, when Dr. Sharemuch finally arrived at the conference, Dr. Analysis II was waiting for him with a cup of fresh coffee and the news of her discovery. After a few cups and two delicious slices of cake, Dr. Sharemuch was delighted. To see someone so eager to make use of his open data and code was a joy, and he invited Dr. Analysis II to collaborate on a new project investigating bilinguals' risk-taking compared to monolinguals. Dr. Analysis II was overwhelmed with excitement. Who would have thought that the simple act of sharing a data spreadsheet would be the key to solving mysteries, unlocking amazing discoveries, and embarking on new collaborative adventures?

\section{An epic tale of large-scale collaboration}

Dr. Small-Town had a destiny, revealed to him in an ancient text passed down from his grandmother: to bring knowledge to his town of Elbit. Elbit was a scarcely populated area with two prominent populations, elves and hobbits. Some families had roots in both lineages and spoke both Elvish and Hobbitish at home. Dr. Small-Town was deeply interested in bilingualism

294 and strove to recruit every bilingual he could track down, to broaden his knowledge of the 295 Elbitian people and fulfill his destiny. Despite his efforts, he was aware that the statistical 296 inferences he could make with a sample size as small as he had were very limited, and he felt 297 powerless. After sharing his feelings and concerns on an appropriate online forum (i.e., Twitter), 298 he was all but ready to give up on his passion for bilingualism research and study something else. 299 Just then, a tweet appeared on his timeline, sharing a large-scale collaboration being done by 
infant bilingualism researchers (Byers-Heinlein et al., 2021). Against all odds, Dr. Small-Town felt his heart beating again: there was hope!

Clicking the link in the tweet, Dr. Small-Town learned that large-scale collaborations could be an effective solution to the problem of small samples. He knew that this could be just the thing for him to make a contribution to bilingualism research! In the following weeks, he joined forces with other labs (who had access not only to elves and hobbits, but also centaurs and dragons), and together with researchers from these labs, they drafted a solid, pre-registered research protocol. In the next few months, Dr. Small-Town followed the protocol as closely as possible and collected data with as many Elbitian bilinguals as he could find, resulting in drumroll please - 17 participants! Finally, data from all the labs were pooled and analyzed using a single analytic framework. With such combined statistical power, sophisticated modeling was possible, and Dr. Small-Town was able to draw some exciting conclusions about bilinguals. But it wasn't just the unexpected and nuanced results that excited Dr. Small-Town. By collaborating with researchers from diverse locations and backgrounds, he learned a great deal about the importance of diversity in research, with respect to both researchers and participants. For example, researchers in centaur-dominant areas, which tended to be less advantaged, faced bigger hurdles in participating in the collaboration, due to the lack of materials translated into Centaurish and older lab equipment. The consortium of labs taking part in the collaboration pooled together their resources to help pay a translator and upgrade the centaurs' equipment, so that all labs could take part as much as possible. Participants were also affected by certain research choices. Dragons seemed to have more difficulty with the demographic questionnaire than other participants, and Dr. Small-Town realized that it might have been due to dragons' cultural norms surrounding wealth and privacy. He thought back to previous studies of his where 
323 there were some confusing answers about socio-economic status and now saw this was due to a

324 lack of understanding of participants' cultural backgrounds and the sensitivity of such

325 information. With his new-found cultural awareness, thanks to the large-scale collaboration, he

326 was able to redesign his questionnaires to be more widely applicable and inclusive.

327 Dr. Small-Town's experience in this large-scale collaboration was also invaluable in

328 another way, for he was getting on in years, and needed to find the next-generation knowledge-

329 seeker to whom he would pass down his ancient prophetic text. He carefully selected a protégée

330 from his keen cohort of undergraduates, and used the lessons learned from his collaboration to

331 introduce the standard methods, materials, and scripts that would make knowledge-generation

332 swifter. His protégée, Holly, was eager to take part in such collaborative projects, which would

333 give her access to real (and huge!) datasets (e.g., Hardwicke et al., 2021) on which she could

334 hone her skills, before her time came to take Dr. Small-Town's place as Chief Knowledge-

335 Seeker. She took her training very seriously and innovated a new way to test even more

336 participants: transforming in-person studies to online versions, building on the standardized

337 methods and measures that were openly shared by the large-scale consortiums. As an old man

338 looking back, Dr. Small-Town is happy that he did not give up on his passion for bilingualism

339 and that he learned how to fulfill his destiny as a knowledge-seeker in an inclusive and impactful

340 way.

341 The story of the adventurous Dr. All-in-One

342 Dr. All-in-One was excited about open science. She knew the advantages of collaborating

343 on large-scale and online projects, pre-registering a study, openly sharing materials, data and

344 analysis code, and posting preprints. She also knew that any additional effort to embrace open

345 science would be worth it in the end. But Dr. All-in-One also felt uneasy. She was on the tenure 
track and adopting these practices would mean changing the way she had done things since her early years in bilingualism research. Her thoughts were spinning in endless loops of worry: If I post a preprint, will a potential reviewer read it and compromise the anonymous review process? Will I need to test hundreds of participants? What about data scooping? If I do a preregistration and find other patterns in my data, will I be able to deviate from my plan? What if there's a mistake in my code? Because of potentially identifying information, I cannot share all my data: does it make sense to share part of it?

These were very reasonable concerns. As if someone had heard her thoughts, a listserv email popped up in her mailbox: an online symposium on open science and bilingualism would take place in the coming weeks, and guess who was on the panel of speakers? Dr. File-Drawer, Dr. Small-Town, Dr. Fortuneteller, Future-Dr. Brouillard, and Dr. Too-Much-Data! Dr. All-inOne registered at once.

At the symposium, Dr. File-Drawer was the first speaker. They explained how preprints make science more accessible, help to reduce publication bias against null results, and work in parallel to the peer-review process. Dr. All-in-One was relieved to hear that Dr. File-Drawer had not had any difficulty having their submitted manuscripts that were also posted as preprints peerreviewed. In one instance, a reviewer had read the preprint, but contacted the editor to let them know and was still allowed to review with that information disclosed. Dr. All-in-One also realized that she already shared her research prior to publication with potential reviewers, at conferences and seminars. This had never gotten in the way of the peer review process before, so she was heartened that publishing preprints wouldn't either.

Next, Dr. Small-Town shared his story of how to deal with his sample size limitations and how to incorporate online testing. Dr. All-in-One felt more at ease. It was now clear that 
369 although testing hundreds of participants can be desirable, it is not the only way to do good

370 science. Open science practices highlight the importance of reproducibility and adequate

371 statistical power, which can be achieved by using more sensitive methods and analyses, studying

372 phenomena with larger effect sizes, and testing more trials per participant - not just huge

373 datasets.

374 While Dr. All-in-One was encouraged by Dr. Small-Town's experience, the symposium 375 continued and started to touch on one of her deepest fears: data scooping. Dr. Fortuneteller began

376 to share his struggles when trying to publish his most peculiar research on bilinguals being better 377 than monolinguals at walking backwards. After hearing Dr. Fortuneteller's story, Dr. All-in-One 378 recognized how pre-registration (peer reviewed via a Registered Report or not) might have 379 prevented all those publishing struggles. Dr. Fortuneteller concluded his talk with a discussion of 380 how pre-registering a study decreases the chances of data being scooped. While open science 381 repositories allow embargos that can serve as protective mechanisms, having a pre-registration 382 also serves as a time-stamped public proof of idea, methods, and analysis plan conceptualization 383 - which by no means limits exploratory data analyses.

Future-Dr. Brouillard spoke next on open materials. Dr. All-in-One, being new to coding reproducible analyses, was a little anxious about sharing her materials. She was reassured to hear 386 that Future-Dr. Brouillard had received valuable and constructive feedback from other 387 researchers on her task coding that helped streamline them for future studies and even led to new 388 research questions and collaborations. The session ended with a commentary by the moderator Dr. Too-Much-Data, who 390 discussed the benefits of data sharing. One comment really resonated in Dr. All-in-One's mind: when it comes to sharing, more is usually better, but it is also perfectly fine to start small and 
392 share only the parts that are critical for reproducibility (i.e., to replicate the analyses presented in 393 a paper). Dr. Too-Much-Data also mentioned that in bilingualism research, the language

394 characteristics of the population studied should be shared as a raw dataset with an explanation

395 document, or data dictionary. On the other hand, any personal health information or personal 396 identifier should always be kept private.

397 Dr. All-in-one would still need to figure out the ins and outs for her own research, but 398 after the symposium she was reassured: open science is not impossible - quite the opposite - it is 399 feasible and inclusive. Open science comes in many shapes and forms but always has at its core 400 one common feature: improving science.

$401 \quad$ Final remarks

Many discoveries and invaluable eureka moments await those who bravely venture into

403 bilingualism research. Bilingualism research has the power to contribute to a better

404 understanding of human development, learning, and behavior. But researchers need to be 405 particularly diligent when dealing with the variability inherent to bilingualism. Open science 406 practices are great tools to do so. By embracing transparency, avoiding questionable research 407 practices, striving for reasonable statistical power, and being ready to collaborate and increase 408 diversity, we can build a much stronger science of bilingualism. While each of the open science 409 practices we have discussed also has its downsides and particular challenges (admittedly our 410 stories were optimistic in tone, and we leave further discussion of concerns about embracing 411 open science practices to the technical papers referred to in Table 1), we believe that the benefits 412 of open science strongly outweigh the limitations. Of course, new adventures can bring discomfort, but they also create unique opportunities 414 for innovation and growth. We hope that our stories have resonated with your personal 
415 experiences as a researcher and that, by doing so, they have convinced you that the costs of

416 learning open science practices are easily surpassed by the benefits for individual scientists and

417 for the scientific community. This is the time to band together on the adventure towards open

418 science - will you come? 


\section{References}

Aczel, B., Szaszi, B., Sarafoglou, A., Kekecs, Z., Kucharský, Š., Benjamin, D., Chambers, C. D., Fisher, A., Gelman, A., Gernsbacher, M. A., Ioannidis, J. P., Johnson, E., Jonas, K., Kousta, S., Lilienfeld, S. O., Lindsay, D. S., Morey, C. C., Monafò, M., Newell, B. R., ... Wagenmakers, E.-J. (2019). A consensus-based transparency checklist. Nature Human Behaviour. https://doi.org/10.1038/s41562-019-0772-6

Agnoli, F., Wicherts, J. M., Veldkamp, C. L. S., Albiero, P., \& Cubelli, R. (2017). Questionable research practices among Italian research psychologists. PLOS ONE, 12(3), 1-17. https://doi.org/10.1371/journal.pone.0172792

Allen, C., \& Mehler, D. M. A. (2019). Open science challenges, benefits and tips in early career and beyond. PLOS Biology, 17(12), e3000587. https://doi.org/10.1371/journal.pbio.3000587

Banks, G. C., Field, J. G., Oswald, F. L., O’Boyle, E. H., Landis, R. S., Rupp, D. E., \& Rogelberg, S. G. (2019). Answers to 18 Questions About Open Science Practices. Journal of Business and Psychology, 34(3), 257-270. https://doi.org/10.1007/s10869-018-9547-8

Banks, G. C., Rogelberg, S. G., Woznyj, H. M., Landis, R. S., \& Rupp, D. E. (2016). Editorial: Evidence on Questionable Research Practices: The Good, the Bad, and the Ugly. Journal of Business and Psychology, 31(3), 323-338. https://doi.org/10.1007/s10869-016-9456-7

Bergmann, C., Tsuji, S., Piccinini, P. E., Lewis, M. L., Braginsky, M., Frank, M. C., \& Cristia, A. (2018). Promoting Replicability in Developmental Research Through Meta-analyses: Insights From Language Acquisition Research. Child Development, 89(6), 1996-2009. https://doi.org/10.1111/cdev.13079

Burman, L. E., Reed, W. R., \& Alm, J. (2010). A Call for Replication Studies. Public Finance 
Review, 38(6), 787-793. https://doi.org/10.1177/1091142110385210

Brouillard, M., \& Byers-Heinlein, K. (2021). ADHD and bilingualism in young adults:

Revisiting executive functioning and bilingual vocabulary. Open Science Framework. https:// http://doi.org/10.17605/osf.io/r5kpq

Byers-Heinlein, K., Bergmann, C., Davies, C., Frank, M. C., Hamlin, J. K., Kline, M.,

Kominsky, J. F., Kosie, J. E., Lew-Williams, C., Liu, L., Mastroberardino, M., Singh, L., Waddell, C. P. G., Zettersten, M., \& Soderstrom, M. (2020). Building a collaborative psychological science: Lessons learned from ManyBabies 1. Canadian Psychology/Psychologie Canadienne, 1-51. https://doi.org/10.1037/cap0000216

Byers-Heinlein, K., Tsui, A. S. M., Bergmann, C., Black, A. K., Brown, A., Carbajal, M. J., Durrant, S., Fennell, C. T., Fiévet, A.-C., Frank, M. C., Gampe, A., Gervain, J., GonzalezGomez, N., Hamlin, J. K., Havron, N., Hernik, M., Kerr, S., Killam, H., Klassen, K., ... Wermelinger, S. (2021). A Multilab Study of Bilingual Infants: Exploring the Preference for Infant-Directed Speech. Advances in Methods and Practices in Psychological Science, 4(1), 251524592097462. https://doi.org/10.1177/2515245920974622

Chambers, C.D., \& Tzavella, L. (2021). The past, present and future of Registered Reports. Nature Human Behaviour, 1-14. https://doi.org/10.1038/s41562-021-01193-7

Connor, D. B. O. (2021). Leonardo da Vinci, pre-registration and the architecture of science: Towards a more open and transparent research culture. Health Psychology Bulletin, 1-15.

Crüwell, S., van Doorn, J., Etz, A., Makel, M. C., Moshontz, H., Niebaum, J. C., Orben, A., Parsons, S., \& Schulte-Mecklenbeck, M. (2019). Seven Easy Steps to Open Science. Zeitschrift Für Psychologie, 227(4), 237-248. https://doi.org/10.1027/2151-2604/a000387 Dailey, S., \& Bergelson, E. (2021). Language Input to Infants of Different Socioeconomic 
Statuses: A Quantitative Meta-Analysis. PsyArXiv.

Ebersole, C. R., Mathur, M. B., Baranski, E., Bart-Plange, D.-J., Buttrick, N. R., Chartier, C. R., Corker, K. S., Corley, M., Hartshorne, J. K., IJzerman, H., Lazarević, L. B., Rabagliati, H., Ropovik, I., Aczel, B., Aeschbach, L. F., Andrighetto, L., Arnal, J. D., Arrow, H., Babincak, P., ... Nosek, B. A. (2020). Many Labs 5: Testing Pre-Data-Collection Peer Review as an Intervention to Increase Replicability. Advances in Methods and Practices in Psychological Science, 251524592095868. https://doi.org/10.1177/2515245920958687

Frank, M. C., Bergelson, E., Bergmann, C., Cristia, A., Floccia, C., Gervain, J., Hamlin, J. K., Hannon, E. E., Kline, M., Levelt, C., Lew-Williams, C., Nazzi, T., Panneton, R., Rabagliati, H., Soderstrom, M., Sullivan, J., Waxman, S., \& Yurovsky, D. (2017). A Collaborative Approach to Infant Research: Promoting Reproducibility, Best Practices, and TheoryBuilding. Infancy, 22(4), 421-435. https://doi.org/10.1111/infa.12182

Frankenhuis, W. E., \& Nettle, D. (2018). Open Science Is Liberating and Can Foster Creativity. Perspectives on Psychological Science, 13(4), 439-447. https://doi.org/10.1177/1745691618767878

Hales, A. H., Wesselmann, E. D., \& Hilgard, J. (2019). Improving Psychological Science through Transparency and Openness: An Overview. Perspectives on Behavior Science, 42(1), 13-31. https://doi.org/10.1007/s40614-018-00186-8

Hardwicke, T. E., Bohn, M., MacDonald, K., Hembacher, E., Nuijten, M. B., Peloquin, B. N., DeMayo, B. E., Long, B., Yoon, E. J., \& Frank, M. C. (2021). Analytic reproducibility in articles receiving open data badges at the journal Psychological Science : an observational study. Royal Society Open Science, 8(1), 201494. https://doi.org/10.1098/rsos.201494

Hardwicke, T. E., Mathur, M. B., MacDonald, K., Nilsonne, G., Banks, G. C., Kidwell, M. C., 
Hofelich Mohr, A., Clayton, E., Yoon, E. J., Henry Tessler, M., Lenne, R. L., Altman, S., Long, B., \& Frank, M. C. (2018). Data availability, reusability, and analytic reproducibility: evaluating the impact of a mandatory open data policy at the journal Cognition. Royal Society Open Science, 5(8), 180448. https://doi.org/10.1098/rsos.180448

Havron, N., Bergmann, C., \& Tsuji, S. (2020). Preregistration in infant research - A primer. Infancy, 25(5), 734-754. https://doi.org/10.1111/infa.12353

Ioannidis, J. P. A. (2005). Why Most Published Research Findings Are False. PLoS Medicine, 2(8), e124. https://doi.org/10.1371/journal.pmed.0020124

Kerr, N. L. (1998). HARKing: Hypothesizing after the results are known. Personality and social psychology review, 2(3), 196-217. https://doi.org/10.1207/s15327957pspr0203_4

Joel, S., Eastwick, P. W., \& Finkel, E. J. (2018). Open Sharing of Data on Close Relationships and Other Sensitive Social Psychological Topics: Challenges, Tools, and Future Directions. Advances in Methods and Practices in Psychological Science, 1(1), 86-94. https://doi.org/10.1177/2515245917744281

John, L. K., Loewenstein, G., \& Prelec, D. (2012). Measuring the Prevalence of Questionable Research Practices With Incentives for Truth Telling. Psychological Science, 23(5), 524532. https://doi.org/10.1177/0956797611430953

Kathawalla, U.-K., Silverstein, P., \& Syed, M. (2020). Easing Into Open Science: A Tutorial for Graduate Students. PsyArXiv, 1-31.

Klein, R. A., \& Al., E. (2013). Many Labs 4: Failure to Replicate Mortality Salience Effect With and Without Original Author Involvement. 53(9), 1689-1699. 
509 Kremin, L. V., \& Byers-Heinlein, K. (2021). Why not both? Rethinking categorical and

510

511

512

513

514

515

516

517

518

519

520

521

522

523

524

525

526

527

528

529

530

531 continuous approaches to bilingualism. International Journal of Bilingualism, 13670069211031986. https://doi.org/10.1177/13670069211031986

Leivada, E., Westergaard, M., Duñabeitia, J. A., \& Rothman, J. (2020). On the phantom-like appearance of bilingualism effects on neurocognition: (How) should we proceed? Bilingualism: Language and Cognition, 1-14. https://doi.org/10.1017/S1366728920000358

Lewis, M., Mathur, M. B., VanderWeele, T. J., \& Frank, M. C. (2020). The puzzling relationship between multi-lab replications and meta-analyses of the published literature. PsyArXiv, 117.

Lindsay, D. S. (2020). Seven steps toward transparency and replicability in psychological science. Canadian Psychology/Psychologie Canadienne, 61(4), 310-317. https://doi.org/10.1037/cap0000222

Marian, V., \& Hayakawa, S. (2020). Measuring bilingualism: The quest for a "bilingualism quotient." Applied Psycholinguistics, 1-22. https://doi.org/10.1017/S0142716420000533

McKiernan, E. C., Bourne, P. E., Brown, C. T., Buck, S., Kenall, A., Lin, J., McDougall, D., Nosek, B. A., Ram, K., Soderberg, C. K., Spies, J. R., Thaney, K., Updegrove, A., Woo, K. H., \& Yarkoni, T. (2016). How open science helps researchers succeed. ELife, 5(JULY), 119. https://doi.org/10.7554/eLife. 16800

Mertzen, D., Lago, S., \& Vasishth, S. (2021). The benefits of preregistration for hypothesisdriven bilingualism research. Bilingualism: Language and Cognition, 1-6. https://doi.org/10.1017/S1366728921000031

Meyer, M. N. (2018). Practical Tips for Ethical Data Sharing. Advances in Methods and Practices in Psychological Science, 1(1), 131-144. 
https://doi.org/10.1177/2515245917747656

Munafò, M. R., Nosek, B. A., Bishop, D. V. M., Button, K. S., Chambers, C. D., Percie du Sert, N., Simonsohn, U., Wagenmakers, E.-J., Ware, J. J., \& Ioannidis, J. P. A. (2017). A manifesto for reproducible science. Nature Human Behaviour, 1(1), 0021. https://doi.org/10.1038/s41562-016-0021

Murphy, M. C., Mejia, A. F., Mejia, J., Yan, X., Cheryan, S., Dasgupta, N., Destin, M., Fryberg, S. A., Garcia, J. A., Haines, E. L., Harackiewicz, J. M., Ledgerwood, A., Moss-Racusin, C. A., Park, L. E., Perry, S. P., Ratliff, K. A., Rattan, A., Sanchez, D. T., Savani, K., ... Pestilli, F. (2020). Open science, communal culture, and women's participation in the movement to improve science. Proceedings of the National Academy of Sciences of the United States of America, 117(39), 24154-24164. https://doi.org/10.1073/pnas.1921320117

Nelson, L. D., Simmons, J., \& Simonsohn, U. (2018). Psychology’s Renaissance. Annual Review of Psychology, 69(1), 511-534. https://doi.org/10.1146/annurev-psych-122216-011836

Nosek, B. A., Alter, G., Banks, G. C., Borsboom, D., Bowman, S. D., Breckler, S. J., Buck, S., Chambers, C. D., Chin, G., Christensen, G., Contestabile, M., Dafoe, A., Eich, E., Freese, J., Glennerster, R., Goroff, D., Green, D. P., Hesse, B., Humphreys, M., ... Yarkoni, T. (2015). Promoting an open research culture. Science, 348(6242), 1422-1425. https://doi.org/10.1126/science.aab2374

Nosek, Brian A., Ebersole, C. R., DeHaven, A. C., \& Mellor, D. T. (2018). The preregistration revolution. Proceedings of the National Academy of Sciences, 115(11), 2600-2606. https://doi.org/10.1073/pnas.1708274114

Nosek, Brian A., \& Errington, T. M. (2020). The best time to argue about what a replication means? Before you do it. Nature, 583(7817), 518-520. https://doi.org/10.1038/d41586-020- 
02142-6

Open Science Collaboration. (2015). Estimating the reproducibility of psychological science. Science, 349(6251), aac4716-aac4716. https://doi.org/10.1126/science.aac4716

Pownall, M. (2020). Pre-registration in the undergraduate dissertation: A critical discussion. Psychology Teaching Review, 26(1), 71-76.

Rubin, M. (2017). When Does HARKing Hurt? Identifying When Different Types of Undisclosed Post Hoc Hypothesizing Harm Scientific Progress. Review of General Psychology, 21(4), 308-320. https://doi.org/10.1037/gpr0000128

Scheel, A. M., Schijen, M., \& Lakens, D. (2021). An excess of positive results: Comparing the standard psychology literature with registered reports. Advances in Methods and Practices in Psychological Science, 1-14.

Scheel, A. M., Tiokhin, L., Isager, P. M., \& Lakens, D. (2020). Why Hypothesis Testers Should Spend Less Time Testing Hypotheses. Perspectives on Psychological Science, 174569162096679. https://doi.org/10.1177/1745691620966795

Sijtsma, K. (2016). Playing with Data - Or How to Discourage Questionable Research Practices and Stimulate Researchers to Do Things Right. Psychometrika, 81(1), 1-15. https://doi.org/10.1007/s11336-015-9446-0

Silberzahn, R., Uhlmann, E. L., Martin, D. P., Anselmi, P., Aust, F., Awtrey, E., Bahník, Š., Bai, F., Bannard, C., Bonnier, E., Carlsson, R., Cheung, F., Christensen, G., Clay, R., Craig, M. A., Dalla Rosa, A., Dam, L., Evans, M. H., Flores Cervantes, I., ... Nosek, B. A. (2018). Many Analysts, One Data Set: Making Transparent How Variations in Analytic Choices Affect Results. Advances in Methods and Practices in Psychological Science, 1(3), 337356. https://doi.org/10.1177/2515245917747646 
578 Simmons, J. P., Nelson, L. D., \& Simonsohn, U. (2011). False-Positive Psychology.

579

580

581

582

583

584

585

586

587

588

589

590

591

592

593

594

595

596

597

598
Psychological Science, 22(11), 1359-1366. https://doi.org/10.1177/0956797611417632

Soderberg, C. K., Errington, T. M., Schiavone, S. R., Bottesini, J., Thorn, F. S., Vazire, S., Esterling, K. M., \& Nosek, B. A. (2020). Initial Evidence of Research Quality of Registered Reports Compared to the Standard Publishing Model. MetaArXiv, 1-59.

Surrain, S., \& Luk, G. (2019). Describing bilinguals: A systematic review of labels and descriptions used in the literature between 2005-2015. Bilingualism: Language and Cognition, 22(2), 401-415. https://doi.org/10.1017/S1366728917000682

Syed, M., \& Kathawalla, U.-K. (2020). Cultural Psychology, Diversity, and Representation in Open Science. August, 1-23. https://doi.org/10.31234/osf.io/t7hp2

Tsou, A., Schickore, J., \& Sugimoto, C. R. (2014). Unpublishable research: examining and organizing the "file drawer." Learned Publishing, 27(4), 253-258. https://doi.org/10.1087/20140404

Wicherts, J. M., Veldkamp, C. L. S., Augusteijn, H. E. M., Bakker, M., van Aert, R. C. M., \& van Assen, M. A. L. M. (2016). Degrees of Freedom in Planning, Running, Analyzing, and Reporting Psychological Studies: A Checklist to Avoid p-Hacking. Frontiers in Psychology, 7(NOV), 1-12. https://doi.org/10.3389/fpsyg.2016.01832

Wu, L., Wang, D., \& Evans, J. A. (2019). Large teams develop and small teams disrupt science and technology. Nature, 566(7744), 378-382. https://doi.org/10.1038/s41586-019-0941-9

Yamada, Y. (2018). How to Crack Pre-registration: Toward Transparent and Open Science. Frontiers in Psychology, 9(SEP), 1-3. https://doi.org/10.3389/fpsyg.2018.01831 